\title{
Merkblatt Wichtige Geräteeinstellungen
}

\section{B- Bild/Grauwertbild}

1. Sonde Für geplante Untersuchung geeignete Sonde wählen (Convex, Linear, Sektor, Hockey, Vaginal, usw.) auf Sonde oft Frequenzbereich angegeben (als Sondenname) z. B. C 5, L 9, usw.

2. Preset Für diese Untersuchung geeignetes Preset/ Voreinstellung/ Programm wählen (Abdomen, Schilddrüse, Karotis, Schulter, small Parts, Mamma, usw.).

Diese sind nicht fix, sondern individuell veränder-/programmier-/ benenn- und speicherbar.

Mit Applikationsspezialist/in Presets optimal für eigene Bedürfnisse festlegen und benennen!

3. Monitor An jedem sind Helligkeit und Kontrast verstellbar (LCD- Displays sind in der Darstellung stabiler).
Folgende Einstellungen kontinuierlich optimieren!

das heisst: mit rechter (linker) Hand Sonde führen, linke Hand (rechte) konstant an den Einstellknöpfen! Beim „Ausprobieren“ Knöpfe voll durchdrehen (-kippen), da Effekt und Bereich bei verschiedenen Geräten/Firmen unterschiedlich: Möglichkeiten des (eigenen) Gerätes kennenlernen!

Eventuell anfangs (Kurse, Anfänger) wichtigste Knöpfe mit roten Klebpunkten markieren.

4. Gesamtverstärkung $=$ Helligkeit des Grauwert-/ B- Bildes

Gain/ Main- Gain: Ganzes Ultraschallbild wird heller oder dunkler.

5. Stufengain = tiefenselektive Verstärkung/ Helligkeitsausgleich

TGC oder DGC = Time or Depth Gain Compensation: entsprechender Bildbereich wird heller oder dunkler. Schieberreihe, meist rechts oder links oben auf Keyboard.

6. Eindringtiefe = Untersuchungs- bzw. Bildtiefe

Tiefe/ Depth. Am Bildrand mit Strichen, oft auch in $\mathrm{cm}$ angegeben

$\rightarrow$ Untersuchungsbereich bildfüllend einstellen

Zoom/ RES = Vergrösserung. Je nach Gerät verschieden:

Nur Spreizung oder echte Fokussierung auf Bildausschnitt, dieser mit scrollen verschiebbar.

7. Fokusposition = Bereich der besten Ortsauflösung = Bildschärfe Ein oder mehrere Pfeile am Bildrand Fokus/ Fokuszone/ Scharfstellung/ Nahfeld/ Fernfeld

Ein oder mehrere Fokuszonen wählbar (je mehr Foci, je tiefer die Bildfrequenz = Bil- 
der $/ \mathrm{sec}=$ frame rate $=$ FR). Für Gesamtbild im unteren Bilddrittel einstellen.

Für Spezielles Fokus auf untersuchten Bereich einstellen.

\section{Frequenz = Sendefrequenz in $\mathrm{MHz}$} Bei allen neueren Geräten verstellbar, immer auf Bild angegeben

Abdomen- Sonden: Bereich 2-6 MHz

Small-Part- Sonden: Bereich (5) 7 - 15 (18) $\mathrm{MHz}$

Bei Breitband- Sonden Frequenzgewichtung verstellbar (Penetration = tief, General, Resolution $=$ hoch)

Für Oberflächliches: möglichst hohe Frequenz (wenig Tiefe, Bild feiner)

Für grössere Tiefe (dicke Patienten): tiefe
Frequenz (viel Tiefe, Bild gröber)

Tissue -(second) Harmonic Imaging (T oder $\mathrm{H}$ vor Frequenzanzeige)

Prinzip: Empfang/ Bildaufbau mit doppelter Sendefrequenz (genial!).

9. Preprocessing Dynamikbereich/ Dynamic Range/ DYN/ Enhance (Messgrösse: $\mathrm{dB}=$ Dezibel) Grauwertabstufung verstellbar, in $\mathrm{dB}$ angegeben (ca. 50 - 100), hohe $\mathrm{dB}(80-100)$ ergeben ein weiches, tiefe $\mathrm{dB}$ $(50-60)$ ein kontrastreiches Bild > schwarzweiss.

10. Je nach Gerät / Firma viele weitere Optionen/ Schalter/ Knöpfe Suchen! Ausprobieren! $\rightarrow$ tolle Überraschungen.
PS Alle neueren Geräte haben einen so genannten Optimierungsknopf (verschieden benannt, fragen).

Meist optimiert dieser nur die Helligkeit und den Helligkeitsausgleich (4. und 5.), alles andere nicht!

oder spreizt den Grauwertbereich auf die im momentanen Bild verwendeten Werte (oder? fragen).

Viel Erfolg und viel Vergnügen

Jürg Prim

Siehe auch: http://www.sgum.ch/ support/tricks_tipps/tricks_tipps.htm 